\title{
Surgical removal of an undifferentiated abdominal sarcoma from a koi carp (Cyprinus carpio)
}

\begin{abstract}
G. A. Lewbart, G. Spodnick, N. Barlow, N. E. Love, F. Geoly, R. S. Bakal
Veterinary Record (1998) 143, 556-558

An undifferentiated abdominal sarcoma was removed surgically from a koi carp. The diagnostic procedures, including radiography and computed tomography, and the procedures for general anaesthesia and the surgical approach for a celiotomy in a fish are described. The gross and microscopic appearance of the tumour is described and illustrated.

of the coelomic cavity. The mass was central and to the left of the midline. The gastrointestinal tract was displaced caudally and to the right (Fig 1). The mass had multifocal regions of hypoattenuation and mineralisation (Figs 1 and 2). It was considered that the mass might originate in the liver, gonads, or less likely the gastrointestinal tract or spleen. Neoplasia with associated tumour necrosis, granuloma and abscess were differential diagnoses.
\end{abstract}

A THREE-YEAR-OLD mature male showa koi (Cyprinus carpio), weighing $1792 \mathrm{~g}$, had had a distended abdomen for two months. The fish had been fed a diet of commercially prepared koi pellets, and the water quality on the fish farm where it lived was within normal limits.

\section{Materials and methods}

Radiographic examination, computed tomography and ultrasound

Left to right lateral and right and left lateral decubital (horizontal beam) whole body radiographs, caudal coelomic cavity ultrasonography and computed tomography (CT) were used to characterise the coelomic mass and localise the organ of origin.

The fish was placed directly upon a plastic-covered film cassette (Dupont Ultravision UV-G; EI duPont deNemours) to obtain the right lateral view and on a plastic-covered platform for the right and left lateral decubital views. A large, lobulated central to right-sided soft tissue mass with focal faint mineralisation was visible throughout the length of the ventral coelomic cavity. The mass caused focal dorsal displacement of the caudal compartment of the swim bladder. The mass extended to the right of the midline on the right lateral decubital view and caused leftward displacement of the cranial compartment of the swim bladder on the left lateral view. It was considered that the mass might originate in the liver, gonads, gastrointestinal tract, or spleen.

An ultrasound examination (Acuson) was made with the fish out of the water and restrained by the clinician. A 7 and a $5 \mathrm{MHz}$ transducer wrapped in plastic were used for the examination. A mixed echogenic mass with areas of reflective hyperechogenicity (mineralisation) was visible in the central coelomic cavity. The mass was not highly vascular, and differential diagnoses included neoplasia, granuloma, hyperplasia, abscess and, less likely, haematoma.

A non-contrast helical CT (Sytec SRi-General Electric) scan with a pitch of $1(5 / 5)$ was made of the coelomic cavity. The fish was placed directly on the CT gantry in right lateral recumbency. The scan took less than four minutes, the maximum time for which the authors consider that a koi can remain out of water without respiratory assistance. A large mass encompassing the ventral coelomic cavity was identified. It extended from immediately caudal to the cardiac silhouette to the most caudal aspect

G. A. Lewbart, MS, VMD, G. Spodnick, DVM, DipACVS, R. S. Bakal, DVM, MS, Department of Companion Animal and Special Species, Medicine, $\mathbf{N}$. Barlow, DVM, F. Geoly, DVM, DipACVP, Department of Microbiology, Physiology and Pathology, N. E. Love, DVM, DipACVR, Department of Anatomy, Physiological Sciences and Radiology, North Carolina State University, College of Veterinary Medicine, 4700 Hillsborough Street, Raleigh, North Carolina 27606, USA

Dr Spodnick's present address is Veterinary Specialty Hospital, 305-C Asheville Avenue, Cary, North Carolina 27511, USA

Dr Geoly's present address is Heska Corporation, 1825 Sharp Point Drive, Fort Collins, Colorado 80525, USA

\section{Anaesthesia}

The fish was anaesthetised for an exploratory celiotomy. Anaesthesia was induced by immersing the fish in water containing $200 \mathrm{mg}$ tricaine methanesulphonate/litre for five minutes until it had only mild opercular movements (the opercula could be observed moving but the motion was not sufficient to generate water flow across the gills). The fish was placed on the surgery area and the delivery tubes of a recirculating anaesthesia machine were positioned in its mouth. A stock solution of tricaine methanesulphonate was prepared by dissolving $10 \mathrm{~g}$ Finquel (Argent Chemical Labs) in 1 litre of deionised water and buffering it to $\mathrm{pH} 7.0$ with sodium bicarbonate. Anaesthesia was maintained with a solution of $160 \mathrm{mg} /$ litre tricaine methanesulphonate circulated at a flow rate of 3 litres/minute for 55 minutes. The concentration of tricaine methanesulphonate was gradually reduced to 38 $\mathrm{mg} /$ litre over the next 40 minutes as the surgical procedure was being completed. The total duration of anaesthesia was $100 \mathrm{~min}$ utes. The fish was also given an intraperitoneal dose of enrofloxacin $(10 \mathrm{mg} / \mathrm{kg})$ during surgery to reduce the possibility of a secondary bacterial infection. Butorphanol at a dose of 0.1 $\mathrm{mg} / \mathrm{kg}$ was given subcutaneously at the end of the surgery to reduce the sensation of pain.

\section{Surgical procedure}

The scales were removed from the area of the incision site with forceps. A ventral midline abdominal incision was made, beginning immediately caudal to the base of the pectoral fins and extending to within $1 \mathrm{~cm}$ of the cloaca. An osteotome was used to separate the pelvic girdle along its midline. Gelpi perineal retractors were used to keep the coelomic incision open. There was a large solid mass in the abdominal coelom which extended caudally from the liver to the pelvic inlet, and was compressing the internal organs dorsally and to the left. Sharp and blunt dissection were used to free the mass from its attachments to the liver, body wall and kidneys. Larger blood vessels supplying the mass were divided and ligated with 4-0 polypropylene suture (Prolene; Ethicon) and bipolar cautery was used to electrocoagulate the smaller vascular pedicles. The pelvic osteotomy was repaired with 2-0 stainless steel cerclage wires. The muscle wall was closed with 3-0 polyglyconate (Maxon; Davis \& Geck) in a simple continuous pattern and the skin was closed with 4-0 polypropylene in a continuous Ford

\section{Postoperative course}

The fish recovered from the anaesthesia and surgical procedure without complications. The air space in the coelomic cavity caused the fish to be positively buoyant so $110 \mathrm{ml}$ of air was aspirated from the abdomen to correct the problem. The skin sutures were removed 25 days after the surgery. interlocking pattern. 


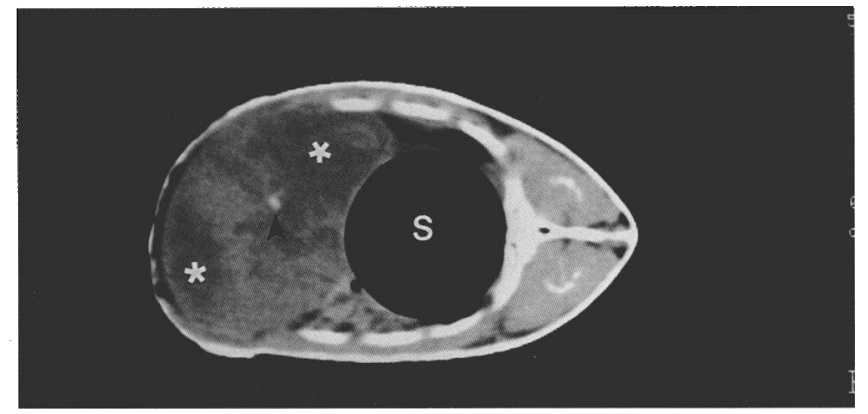

FIG 1: Non-contrast cross-sectional computed tomography image of the coelomic mass. The fish is in right lateral recumbency, the swim bladder (S) is located dorsally. A lefit-sided ventrally located complex soft tissue mass with regions of hypoattenuation $(*)$ and mineralisation (arrowhead) is visible

\section{Pathology}

Grossly, the neoplasm was firm, white to light tan, and lobulated with a thin, orange capsule over several of the sections (Fig 3). There were multiple dark red, depressed areas interpreted to be tumour necrosis. Histologically, the neoplasm was densely cellular, well circumscribed, and partially encapsulated by a thin band of fibrous connective tissue. No adjacent normal tissue was present either grossly or microscopically. The neoplastic cells were highly pleomorphic and arranged in numerous different patterns. Most of the tumour was composed of broad bands and intersecting bundles of closely packed spindle cells with minimal intervening fibrous stroma (Fig 4). In other areas, small closely packed round cells formed islands and cords separated by fibrous septae. The nuclei were large, pleomorphic, and round to oval with granular chromatin. Scattered throughout the spindle-cell areas were small numbers of large, multinucleate cells with intensely eosinophilic cytoplasm. The mitotic rate was three to four mitotic figures per 10 high power fields $(x 400)$. There were large areas of tumour necrosis. The neoplasm invaded the capsule multifocally, closely abutted numerous blood and lymphatic vessels, and was occasionally present within capsular blood vessels (surrounded by erythrocytes) and lymphatic vessels. Multifocally, the capsule contained cystic spaces of varying size lined by a single layer of ciliated cuboidal or flattened epithelium.

The immunohistochemical staining properties of the neoplasm did not help to produce a definitive diagnosis because the multiple antibodies used did not stain appropriate control tissues from a normal koi. Electron microscopy was inconclusive owing to the lack of definitively identifiable structures within the sections examined. The lack of immunohistochemical staining with multiple antibodies and the inconclusive ultrastructural examination ultimately led to a diagnosis of poorly differentiated sarcoma.

\section{Discussion}

Sarcomas are relatively common tumours of fish and there are several reports describing a variety of sarcomas (MawdesleyThomas 1975, Hubbard 1985, Endo 1991, Probasco and others

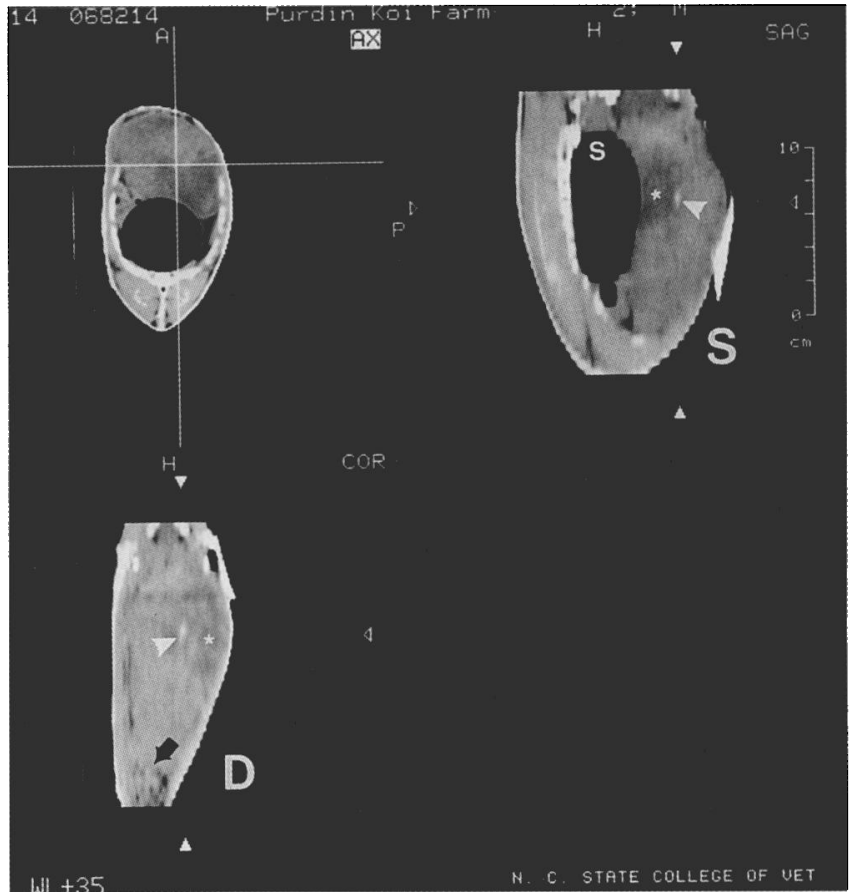

FIG 2: Dorsal (D) and sagittal (S) reformatted computed tomography images of the coelomic mass. In both views, the head of the fish is directed towards the top of the image. The mass is displacing the gastrointestinal tract to the right (black arrow); regions of hypoattenuation $(*)$ and mineralisation (arrowhead) are visible

1994). One detailed report describes gonadal neoplasms of goldfish (Carassius auratus) and carp of the Great Lakes of North America (Down and Leatherland 1989). There are also several descriptions of differentiated and undifferentiated abdominal sarcomas of koi (Crisp 1854, Ronca 1914, Schlumberger and Lucke 1948, Wellings 1969) and papillomas, squamous cell carcinomas, and a branchioblastoma have all been described in koi in recent years (Wildgoose 1992, Wildgoose and Bucke 1995). The authors believe that this is the first report of the successful surgical removal of an abdominal sarcoma from a koi.

The extent of the abdominal incision was determined by the size of the mass. The incision initially extended to just cranial to the pelvic girdle; however, greater caudal exposure for the excision of the mass necessitated an osteotomy of the pelvis. The pelvis appeared to heal without complication and the fish did not experience any ill effects as a result of the osteotomy. The use of bipolar cautery during surgery proved to be invaluable in controlling haemorrhage. Air should have been removed from the abdominal coelom by suction when the muscular layer was closed, to avoid the need to remove the air by abdominocentesis after surgery.

Tricaine methanesulphonate is an FDA-approved and widely used anaesthetic for fishes (Stoskopf 1995). Most fish experience an excitatory stage of anaesthesia, followed by sedation, a loss of equilibrium and finally a loss of any reactivity; it is desirable to maintain anaesthesia at this level. Mild opercular movements should be maintained and the gills must be bathed sufficiently with anaesthetic water. The anaesthetic effects of tricaine
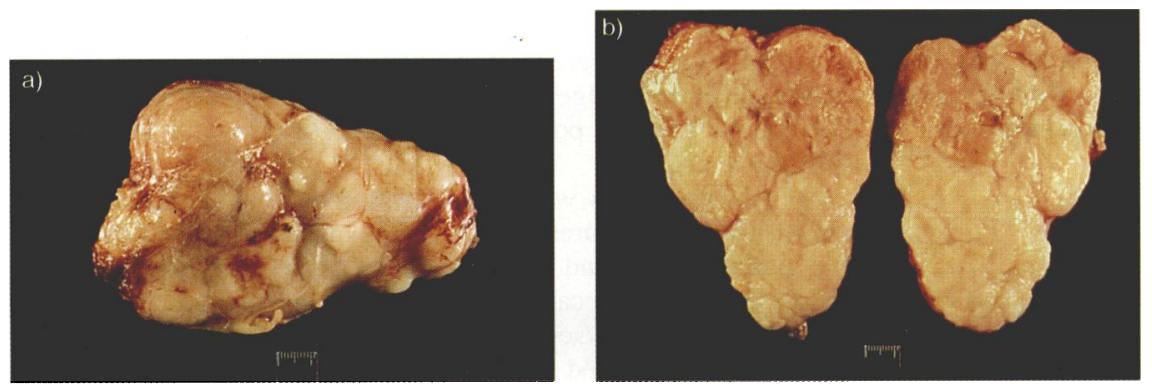

FIG 3: a) Gross view of the tumour. (b) Same view following resection 


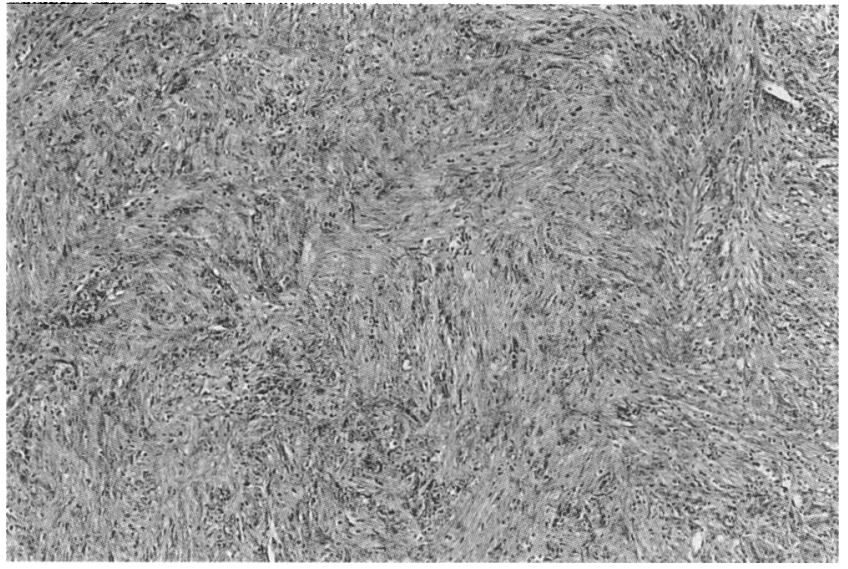

FIG 4: Neoplastic cells separated by a small to moderate amount of fibrovascular stroma. Haematoxylin and eosin $x 132$

methanesulphonate can be quickly reversed by diluting the concentration and placing the fish in fresh, clean water.

The surgical removal of this tumour relieved unnatural pressure on the internal organs and the body wall and corrected the skin ulcer condition. The fish was clinically normal 15 months after surgery.

Acknowledgements. - The authors thank Dr Michael Stoskopf and Dr Craig Harms for their assistance with case management, Dr Ann Kwock for assisting with the interpretation of the clinical pathology samples, and the NCSU-CVM Biomedical Communications Department for photographic assistance.

\section{References}

CRISP, E. (1854) Transactions of the Pathological Society of London 5, 347. (Cited by Wellings 1969)

DOWN, N. E. \& LEATHERLAND, J. F. (1989) Journal of Fish Diseases 12, 415

ENDO, M. (1991) Japanese Journal of Ichthyology 37, 421

HUBBARD, G. B. (1985) Journal of Wildlife Diseases 21, 72

MAWDESLEY-THOMAS, L. E. (1975) The Pathology of Fishes. Eds W. E. Ribelin, G. Migaki. Madison, University of Wisconsin Press. p 805

PROBASCO, D., NOGA, E. \& KHOO, L. (1994) Journal of Small Exotic Animal Medicine 2, 173

RONCA, V. (1914) Tumori 4, 61. (Cited by Wellings 1969).

SCHLUMBERGER, H. G. \& LUCKE, B. (1948) Cancer Research 8, 657

STOSKOPF, M. K. (1995) Current Veterinary Therapy XII. Eds R. W. Kirk, J. D. Bonagura. Philadelphia, W. B. Saunders Col. p 1365

WELLINGS, S. R. (1969) A Symposium on Neoplasms and Related Disorders of Invertebrate and Lower Vertebrate Animals. Eds C. J. Dawe, J. C. Harshbarger. National Cancer Institute Monograph 31. Bethesda, Maryland, USA. p 59

WILDGOOSE, W. H. (1992) Veterinary Record 130, 153

WILDGOOSE, W. H. \& BUCKE, D. (1995) Veterinary Record 136, 418

\title{
Efficacy of a 2 per cent moxidectin gel against gastrointestinal parasites of ponies
}

\author{
C. Bauer, V. Y. Çirak, C. Hermosilla, H. Okoro
}

Veterinary Record (1998) 143, 558-561

The efficacy of moxidectin was evaluated in young ponies naturally infected with gastrointestinal parasites. Eight animals were treated orally with moxidectin at $0.4 \mathrm{mg} / \mathrm{kg}$ bodyweight and eight received only the vehicle. They were all necropsied two weeks later. Faecal samples were examined daily for egg counts and larval cultures. Parasites were recovered from total faecal samples collected daily and from the gastrointestinal tracts at necropsy. Moxidectin reduced the strongyle egg counts by $>99$ per cent from three days after treatment but some individuals remained positive for 10 days. The drug had little or no ovicidal activity. As evaluated in the critical-controlled test, moxidectin was 99 to 100 per cent effective $(P<0.05)$ against luminal stages of Parascaris equorum, Strongylus vulgaris, Triodontophorus species, Craterostomum acuticaudatum, 19 cyathostome species and Oxyuris equi. Adult $S$ edentatus were also completely removed. Its efficacy against third stage larvae of Gasterophilus intestinalis was 95 per cent $(P<0 \cdot 05)$. Luminal nematode stages were removed within a few days, and bots continued to be eliminated for at least two weeks after treatment. No activity was observed against Anoplocephala perfoliata. As evaluated in the controlled test, moxidectin was $\mathbf{1 0 0}$ per cent effective against Habronema muscae $(\mathbf{P}<0 \cdot 05)$ and had a 76 per cent but not significant efficacy against encysted small strongyle larvae.

C. Bauer, DrMedVet, V. Y. Çirak, DrMedVet, C. Hermosilla, DrMedVet, H. Okoro, DVM, MVSc, Institute of Parasitology, Justus Liebig University Giessen, Rudolf-Buchheim-Strasse 2, D-35392 Giessen, Germany
MOXIDECTIN (Fort Dodge) is a macrocyclic lactone with a high activity against adult and larval gastrointestinal parasites of horses and ponies (Lyons and others 1992, Reinemeyer and Aguilar 1992, DiPietro and others 1993, Bello and Laningham 1994, Monahan and others 1995a, b, Solari Basano and Genchi 1996). However, little is known about for how long after the administration of moxidectin the intestinal parasites are removed and the shedding of eggs is reduced. The present dose confirmation trial was designed with particular attention to these time periods.

\section{Materials and methods}

Sixteen ponies of both sexes, one to two-and-a-half years of age, were purchased from three enterprises (two, three and 11 animals), in Eastern Lower Saxony, Germany, and housed in the same stable in November. All the animals had been on pasture during the previous summer and autumn with little or no antiparasitic treatment, and were naturally infected with strongyles as shown by faecal examinations. In January, 13 weeks after they were housed the ponies were weighed and allocated to one of two similar groups of eight, on the basis of source, faecal egg counts and sex. The treated group $M$ ponies had a mean (sd) bodyweight of 121 (47) $\mathrm{kg}$ and were treated orally with moxidectin ( 2 per cent equine gel; Fort Dodge) at a dose rate of $0.4 \mathrm{mg} / \mathrm{kg}$ bodyweight. The control group C ponies weighed 109 (39) $\mathrm{kg}$ and were given the vehicle only.

Faecal egg counts were made by a modified McMaster technique and larval cultures were prepared on samples from each animal daily, before and after treatment. If no worm eggs were counted, another faecal sample was examined qualitatively by using a zinc chloride/sodium chloride flotation technique. Parasite burdens were assessed according to the guidelines of the World 